\title{
A review of in-patient hand infections
}

\author{
A. R. PHIPPS \& J. BLANSHARD \\ Department of Plastic and Reconstructive Surgery, Queen Mary's University Hospital, \\ Roehampton Lane, London SW15 5PN
}

\section{SUMMARY}

A retrospective review is presented of 64 patients with infections of the hand requiring admission to hospital.

We present an account of the different types of hand infection encountered, together with details of the various aetiologies and microbiological findings where these are available.

The management of hand infections is discussed with reference to the patients in our series, and in terms of the general principles involved.

\section{INTRODUCTION AND MATERIALS}

Compared with acute hand injuries, hand infections are now an uncommon cause of admission to hospital, although this was not the case in the years before the introduction of antibiotics. We have traced the records of 64 patients admitted for the treatment of hand infections to Queen Mary's University Hospital, Roehampton \& St James's Hospital, Balham, in a 7-year period between 1981 and 1987.

The wider incidence of hand infections which do not necessitate in-patient treatment is difficult to estimate: however, a total of 24 such patients were treated without admission to hospital during one recent year in a soft tissue clinic held by a plastic surgeon in the A\&E Department at Roehampton. Of course, an indeterminate number of other patients do not reach our notice, but are managed by their general practitioners or do not seek treatment at all.

For the 64 patients in the present survey, we have reviewed the presumed aetiology of the infection and its management before and after admission to hospital. The available results of bacteriological culture were also extracted for review.

Correspondence: $\operatorname{Mr}$ A. R. Phipps, Department of Plastic and Reconstructive Surgery, St Thomas' Hospital, London SE1 TEH. 


\section{RESULTS}

\section{Aetiology of the infections}

Fifteen $(23 \%)$ of these patients were unable to recall an injury which they could associate with their infection. Fifteen patients $(23 \%)$ reported a preceding laceration on a variety of sharp objects, which varied from the probably quite clean to the obviously contaminated (Table 1 ).

Thirteen patients $(20 \%)$ reported bites or scratches at the site of their infection (Table 1). Cat scratches and bites were the most common injury in this category, and were associated with specific infections; these will be discussed.

The factors identified by the remaining 21 patients are summarized in the lower part of Table 1. They include several outdoor fingerprick injuries, two retained wooden splinters and two crushing injuries. Two patients with burns presented with infections complicating small burns that would not otherwise have required hospital attention; we have not considered the special case of patients with hand infections after more extensive burns necessitating admission to the Regional Burns Unit. Five patients are included who required re-admission for post-operative infections following their discharge from hospital after hand surgery; two had undergone carpal tunnel decompression, two had had Kirschner wires inserted as part of surgery for Dupuytren's contracture, and one had undergone repair of a divided flexor pollicis longus tendon.

Table 1. Antecedent injuries.

\begin{tabular}{lc}
\hline Injuries & No. of patients \\
\hline Lacerations & $15(23 \%)$ \\
glass & 3 \\
knife & 2 \\
drains & 2 \\
shovel & 1 \\
chainsaw & 1 \\
unspecified & 4 \\
Bites \& scratches & $13(20 \%)$ \\
cat bite & 5 \\
cat scratch & 2 \\
dog bite & 3 \\
human bite & 1 \\
insect bite & 2 \\
Gardening/camping & $6(9 \%)$ \\
Splinters & $2(3 \%)$ \\
Crush/blow & $2(3 \%)$ \\
Burn & $2(3 \%)$ \\
? iv drug abuse & $1(2 \%)$ \\
Post-operative & $5(8 \%)$ \\
Others & $3(5 \%)$ \\
\hline
\end{tabular}




\section{Nature and sites of infection}

Left and right hands were affected with equal frequency, though in four patients it was impossible to ascertain from the records which was the affected side. There was a marked predilection for the more radially placed digits, as Table 2 shows. Indeed only two infections were recorded in little fingers, and both were associated with the presence of Kirschner wires. One might surmise that this predisposition for infections on the radial side of the hand reflects the more exploratory use to which these digits are put, and their possibly greater susceptibility to minor injury as a result.

The types of infection encountered are seen in Table 3. About three-fifths of patients presented with clinical evidence of purulent collections in the hand. Tendon sheath infections made up $16 \%$ of the cases (10 patients), and this proportion is similar in a study from the U.S.A. (Glass, 1982); in contrast, however, no thenar or mid-palmar space infections were recorded. Local or spreading cellulitis was present in a quarter of our patients, and three patients $(5 \%)$ had evidence of bone or joint sepsis.

Table 2. Sites of infection.

\begin{tabular}{llll}
\hline Left & \multicolumn{1}{c}{ Right } \\
\hline thumb & 5 & thumb & 2 \\
index & 5 & index & 9 \\
middle & 9 & middle & 5 \\
ring & 2 & ring & 3 \\
little & 1 & little & 1 \\
1st web & 2 & 1st web & 0 \\
2nd web & 1 & 2nd web & 0 \\
3rd web & 0 & 3rd web & 1 \\
4th web & 1 & 4th web & 0 \\
palm/dorsum & 6 & palm/dorsum & 7 \\
\hline
\end{tabular}

Table 3. Types of infection.

\begin{tabular}{lc}
\hline Infection & No. of patients \\
\hline Collections of pus & $39(61 \%)$ \\
tendon sheath & 10 \\
pulp space & 8 \\
web space & 5 \\
Cellulitis & $16(25 \%)$ \\
local & 9 \\
spreading & 7 \\
Bone \& Joint infections & $3(5 \%)$ \\
Foreign materials & $6(9 \%)$ \\
\hline
\end{tabular}




\section{Management before admission}

Thirteen patients $(20 \%)$ were referred within 1 day of the beginning of symptoms. Overall, the mean delay before admission was 16 days; the longest interval recorded was 6 months.

Three patients had previously undergone attempts at surgical drainage prior to their referral. About a third had received antibiotics.

\section{Management after admission}

All 64 patients were treated with dressings and immediate elevation of the affected limb. All but 10 patients $(84 \%)$ were given antibiotics (most commonly with flucloxacillin but often in combination with other drugs, including metronidazole). Nineteen patients $(30 \%)$ were successfully managed by these conservative means alone.

The majority of patients, however, required surgical treatment as described in Table 4. Most of these patients underwent simple drainage, but six with tendon sheath infections had formal irrigation of the sheath as illustrated in Fig. 1.

The mean stay in hospital for all patients studied was five days, with a range from 1 to 21 days. As might be expected, the group of patients who were treated for tendon sheath infections stayed longer, on average 9 days.

The majority of patients in the present series regained full function of the affected hand; however two patients suffered a recurrence of sepsis and 9 experienced prolonged stiffness of the hand, one of whom required surgical tenolysis following treatment of a tendon sheath infection. Minor symptoms such as cold-sensitivity were recorded in only two patients, but the authors' personal experience suggests that this may be an under-representation of their true incidence.

\section{Bacteriological findings}

Positive bacterial culture was obtained from 28 patients $(44 \%)$, and the findings are set out in Table 5. The organism most frequently isolated was Staphylococcus aureus $(27 \%)$, none of which was of a methicillin-resistant strain. This finding

Table 4. Management in hospital $19(30 \%)$ treated conservatively; $45(70 \%)$ treated surgically.

\begin{tabular}{lc}
\hline Treatment & No. of patients \\
\hline Drainage & $38(59 \%)$ \\
Irrigation of sheath & $6(9 \%)$ \\
Curettage & $3(5 \%)$ \\
Desloughing & $2(3 \%)$ \\
Removal of nail & $2(3 \%)$ \\
Removal of foreign body & $2(3 \%)$ \\
Removal of K-wire & $2(3 \%)$ \\
\hline
\end{tabular}




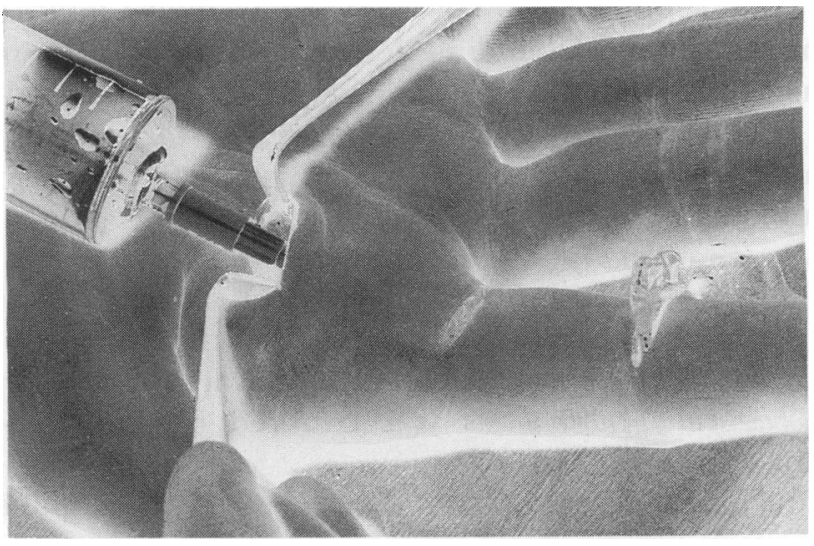

Fig. 1. Irrigation of an infected flexor tendon sheath.

lends a rational basis in retrospect to the use of flucloxacillin as the antibiotic of first choice for these infections.

Pyogenic Streptococci, all of Lancefield group A, were found in three cases (5\%) of diverse aetiology. All of these patients required surgical drainage.

Bite wounds have a reputation for being potentially infected. In the present study Pasteurella multocida was cultured from five hands infected as a result of animal bites or scratches (four by cats, one by a dog). This Gram-negative coccobacillus is a regular finding in the oral cavities of animals ranging from cats, dogs and hamsters to panthers and buffalo (Arons et al., 1982); it is consequently to be expected as a contaminant of bite wounds, but may be missed on culture unless the laboratory request carries the relevant history. Typically, it gives rise to local cellulitis or purulent sepsis, but may progress to septicaemia and meningoencephalitis. It has to date been found to be sensitive to penicillin.

The seven infections resulting from injuries by cats included some of the more serious varieties of infection encountered; they comprised four of the 10 tendon

Table 5. Bacteriological findings.

\begin{tabular}{lc}
\hline Bacteria present & No. of patients \\
\hline Staphylococcus aureus & $17(17 \%)$ \\
$\beta$-haemolytic Streptococcus & $3(5 \%)$ \\
Pasteurella sp & $5(8 \%)$ \\
Mycobacterium tuberculosis & $1(2 \%)$ \\
Enterobacteria & $1(2 \%)$ \\
Candida & $1(2 \%)$ \\
Skin commensals & $5(8 \%)$ \\
No growth & $10(16 \%)$ \\
No report & $25(39 \%)$ \\
\hline
\end{tabular}


sheath infections, one septic arthritis of an interphalangeal joint, one web space infection, and one case of local cellulitis. Canine \& human bites resulted in four localized infections; the three resulting from dog bites all required surgical intervention. Perhaps the tendency of cat bites to give rise to deeply-sited sepsis is a result of the animal's sharply-pointed teeth and penetrating bite, in contrast with the tearing bite inflicted by other species.

Skin commensal organisms were cultured from five wounds, and some other pathogens were each isolated from a single patient. A Candida species was cultured from one paronychial abscess in an infant, who required admission because the patient was too young to tolerate surgical drainage without general anaesthesia. Mycobacterium tuberculosis was cultured from a web-space abscess in an Asian patient.

Anaerobic organisms were not recorded in any of these patients, but it is unlikely that they were specifically sought as a routine.

\section{DISCUSSION}

Hand infections have become a much less frequent indication for surgery than they were before the advent of effective antibiotics: the patients studied here, for example, represent about $0.5 \%$ of all surgical emergencies at Roehampton. However, long-established general principles continue to apply for their successful management.

Superficial hand infections are relatively common and may usually be managed on an out-patient basis if they present early. Small local collections of pus are released under regional anaesthesia. The affected hand is elevated in a high sling and immobilized by local splintage. Regular dressings are instituted. Judicious use is made of antibiotics where indicated, including anti-anaerobic drugs where the history dictates.

Simple infections may still necessitate inpatient management in certain circumstances, such as for surgical drainage in small children who require general anaesthesia, or where the infection complicates a general medical condition such as diabetes.

Neglected or establised infections in the hand always require admission for high elevation and splintage. Clinical suspicion of tendon sheath infection, septic arthritis or infection of the anatomical 'spaces' of the hand dictates urgent surgical exploration. Evident collections of pus also require surgical drainage. Experience suggests that conservative measures are adequate in selected other cases, in which it is reasonable to defer surgery during a trial of conservative treatment. It is valuable, as an aid to monitoring the response to antibiotic therapy, to outline areas of cellulitis on the skin with a marker pen before treatment begins (Fig. 2).

Our bacteriological findings confirm that the majority of positive cultures yield organisms sensitive to flucloxacillin or erythromycin, which are therefore the antibiotics of choice in most hand infections. Although this remains true of infections after bite injuries, we have found that these are more likely to be serious infections requiring vigorous treatment, and we would not question 


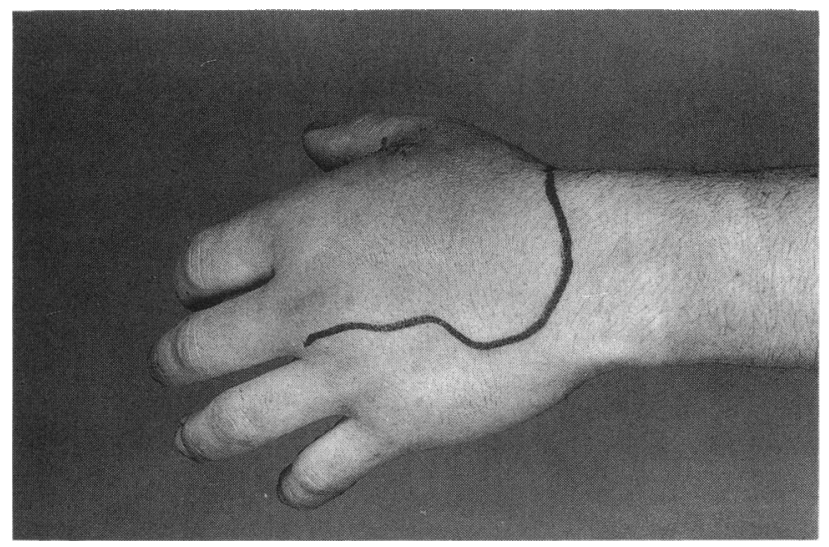

Fig. 2. Marking out the limits of cellulitis before antibiotic treatment.

the wisdom of anti-anaerobic chemotherapy in these cases and early surgical exploration where doubt exists.

\section{ACKNOWLEDGEMENTS}

The authors are grateful to the Consultant Plastic Surgeons of the South West Thames Region for their permission to study patients under their care, and in particular to Mr J. V. Jeffs for his advice and encouragement.

This material was presented at the Winter Meeting of the British Association of Plastic Surgeons held at the Royal College of Surgeons of England in December, 1987.

\section{REFERENCES}

Arons M. S., Fernando L. \& Polayes I. M. (1982) Pasteurella multocida - The major cause of hand infections following domestic animal bites. Journal of Hand Surgery 7, 47-52.

Glass K. D. (1982) Factors related to the resolution of treated hand infections. Journal of Hand Surgery 7, 388-394. 